\title{
Visual error amplification showed no benefit for non-naïve subjects in trunk-arm rowing
}

\author{
Nicolas Gerig ${ }^{1,2,3}{ }^{*}$, Ekin Basalp ${ }^{1}$, Roland Sigrist ${ }^{1}$, Robert Riener ${ }^{1,2}$ \& Peter Wolf ${ }^{1,2}$ \\ 1 Department of Health Science and Technology, ETH Zurich, Zurich, Switzerland \\ 2 Spinal Cord Injury Center at University Hospital Balgrist, Zurich, Switzerland \\ 3 Department of Biomedical Engineering, University of Basel, Basel, Switzerland \\ * Corresponding author: BIROMED-Lab, DBE, University of Basel, Gewerbestrasse 14, 4123 Allschwil, Switzerland \\ Tel: +41 612075470 \\ Email: nicolas.gerig@unibas.ch
}

\section{ORIGINAL ARTICLE}

\section{Article History:}

Submitted $2^{\text {nd }}$ February 2018

Accepted $9^{\text {th }}$ October 2018

Published $4^{\text {th }}$ February 2019

Handling Editor:

Ernst-Joachim Hossner,

University of Bern, Switzerland

Editor-in-Chief:

Martin Kopp

University of Innsbruck, Austria

Reviewers:

Reviewer 1: Hermann Müller University of Giessen, Germany Reviewer 2: Thorsten Stein Karlsruhe Institute of Technology, Germany

\section{ABSTRACT}

Motor learning is assumed to be a partly error driven process. Motor learning studies on simple movements have shown that skilled subjects benefit from training with error amplification. Findings of studies with simple movements do not necessarily transfer to complex sport movements. The goal of this work was to determine the benefit of visual error amplification for non-naïve subjects in learning a fast rowing movement.

We conducted a study comparing non-naïve subjects receiving a fading, visual feedback with visual error amplification against a control group receiving the same visual feedback without error amplification. Separate outcome metrics were applied for the domains of spatial and velocity magnitude errors. Besides error metrics, variability metrics were evaluated for both domains, such that they could be interpreted in quantitative relation to each other.

The implemented error amplification did not cause any beneficial effect. Subjects exposed to error amplification and control did not show different behavior or progress in both error and variability outcomes. Possible reasons remain speculative, e.g. the used error amplification gain could have been wrong. The feasibility of a beneficial error amplification for the training of complex movements remains questionable, since for many required design decisions an informative basis is missing.

Keywords:

Motor Learning - Variability - Error Augmentation - Robot-Assisted Training - Augmented Feedback

Citation:

Gerig, N., Basalp, E., Sigrist, R., Riener, R. \& Wolf P. (2018):Visual error amplification showed no benefit for non-naïve subjects in trunk-arm rowing. Current Issues in Sport Science, 3:013. doi: 10.15203/CISS_2018.013

\section{Introduction}

In conventional training of sports or rehabilitation, a human trainer or therapist can provide augmented feedback on movement execution to the subject. In robot-assisted training, a robot or robotic simulator can also provide feedback. Besides task renderings, i.e. displaying environment and interaction forces that would be naturally present while performing a certain task, a robotic simulator may provide augmented feedback. Augmented feedback from both human trainer or robotic simulator is additional information provided to the trainee to relate his or her individual performance to a desired performance or to an instruction (Sigrist, Rauter, Riener, \& Wolf, 2013).

Augmented feedback can be given both terminally after task execution with the goal to provide better knowledge of results, or concurrently to task execution. Compared to a human trainer, a robot offers more possibilities to record, evaluate, 
augment, and feedback information online, concurrently to task execution. Such concurrent augmented feedback strategies are controversially discussed, have been applied, and have been studied in a variety of different forms (Sigrist et al., 2013).

While human trainers naturally play with refraining, overemphasizing, or providing false augmented feedback to the trainee to make them learn faster, most robotic applied augmented feedback strategies provide true quantitative information to the subject. However, robotic simulators offer numerous possibilities to alter feedback information on performance, movement errors, or even task renderings to interfere with the human trainee's perception. That way, robotic augmented feedbacks might be designed to affect implicit learning processes or to control psychological aspects like motivation or self-efficacy (Duarte \& Reinkensmeyer, 2015). Based on the assumption that motor learning in humans is partly an error driven process, augmenting errors is a promising concept to accelerate or increase learning (Emken \& Reinkensmeyer, 2005; Heuer \& Lüttgen, 2015; Yejun Wei, Bajaj, Scheidt, \& Patton, 2005). Some studies reported beneficial effects of error augmentation (Marchal-Crespo, Schneider, Jaeger, \& Riener, 2014; Rozario, Housman, Kovic, Kenyon, \& Patton, 2009), some studies reported detrimental effects (Bouchard, Corriveau, \& Milot, 2015; Duarte \& Reinkensmeyer, 2015), and sometimes error augmentation was more (MarchalCrespo et al., 2014) or less (Bouchard et al., 2015; Duarte \& Reinkensmeyer, 2015) effective than haptic guidance. A possible explanation for these controversial results is that error augmentation might have overwhelmed the investigated, naïve subjects in the beginning. Being overwhelmed may reduce the subjects' motivation persistently and therefore their training outcome. However, if subjects' motivation is reduced or not also depends on many study specific factors, e.g. task design, rewards, renderings, comfort, or the investigated subject population. These controversial results with naïve subjects exposed to error augmentation may be an indication that error augmentation does not work independent of the subjects' skill level in the investigated task.

Augmenting errors has been reported to be more demanding (Milot, Marchal-Crespo, Green, Cramer, \& Reinkensmeyer, 2010) and to increase task difficulty (Marchal-Crespo, LopezOloriz, Jaeger, \& Riener, 2014), and therefore to be a promising strategy to train skilled subjects, which are non-naïve to the task or already have an advanced level of performance. Indeed, compared to haptic guidance, error augmentation seems to be more effective for more skilled subjects (Marchal-Crespo et al., 2014; Milot et al., 2010). A recent study (Hasson, Zhang, Abe, \& Sternad, 2016) reported that after plateauing, subjects exposed to error augmentation continued to improve their performance in a virtual throwing task, while control subjects did not further improve. The question of such findings from error augmentation strategies translate from such studies in motor learning experiments to training of highly dynamic sports movements remains unanswered.
In our previous studies (Rauter, Sigrist, Riener, \& Wolf, 2015; Sigrist, Rauter, Marchal-Crespo, Riener, \& Wolf, 2014), we investigated augmented feedbacks in training a realistic trunkarm rowing task with a robotic simulator. Learning effects were found from baseline to retention after the first day of training. However, no further learning effects were observed after a second day of training. Given this plateauing of performance after the first day, we wanted to investigate, if exposing subjects to error augmentation on a second day of training would enable them to reach higher performance at the same trunk-arm rowing task.

In motor learning experiments, the term error augmentation is used for both, strategies increasing incidence or amplitude of performed errors, and strategies increasing incidence or amplitude of the perceived errors only. In haptic error augmentation for instance, the robot actively applies forces to cause or amplify movement errors. In visual error augmentation, the displayed error or the rendered position of the avatar, i.e. the virtual representation of the user, on the visual display are distorted. While haptic error augmentation can induce safety risks when performing fast movements with strong robots, visual error augmentation comes with relatively low risks. Visual error augmentation creates a mismatch between visual and proprioceptive information. Such a mismatch could lead to an increased sensory conflict. Sensory conflict is the most commonly assumed driver of cybersickness (LaViola Jr, 2000). Therefore, visual error augmentation could eventually increase incidence or intensity of cybersickness. However, cybersickness or dizziness were never reported by pilot subjects in our rowing simulator. Therefore, we have decided to investigate visual error augmentation.

A typical implementation of error augmentation is augmenting spatial errors only. In reaching movements, the distance from the end-effector to a straight line between start and target position has been used for error augmentation (Celik, Powell, \& Malley, 2009; Patton, Wei, Bajaj, \& Scheidt, 2013; Sharp, Huang, \& Patton, 2011; Y. Wei, Patton, Bajaj, \& Scheidt, 2005). Spatial error augmentation can be applied to arbitrary trajectory tracking movements, e.g. if the spatial error is defined as the distance from the end-effector position to the nearest-neighbor on a reference trajectory. Spatial error is not restricted to end-effector or Cartesian space, but can also be implemented in joint space (Fisher, Huang, Klamroth-Marganska, Riener, \& Patton, 2015). We assumed that a purely spatial error augmentation might be suboptimal if the goal is to teach a movement including temporal aspects or velocity profiles, since temporal errors would not be augmented. As we considered the velocity profile to be an important aspect of our trunk-arm rowing movement, we have decided not to apply a spatial error augmentation only. A common implementation of spatio-temporal error augmentation is using an instantaneous tracking error, e.g. the distance between user position at time $t_{i}$ from reference position at the same time $t_{i}$ (Abdollahi et al., 2013; Rozario et al., 2009). Such a spatio-temporal error augmentation can be implemented visually similar to spatial error augmentation, by 
distorting the displayed avatar in direction of the error, instead of displaying the avatar where it is measured.

The most common visual error augmentation is a linear amplification of the error $\epsilon_{a m p}=\mathrm{p}_{E A} \cdot \epsilon$ with a constant gain $\mathrm{p}_{E A^{\prime}}$ e.g. used in (Celik et al., 2009; Patton et al., 2013; Rozario et al., 2009; Sharp et al., 2011; Wang, Barkana, \& Sarkar, 2010; Y. Wei et al., 2005). For a purely spatial error in two dimensional reaching tasks, measurements with different gains have been performed (Patton et al., 2013; Y. Wei et al., 2005) and efforts to calculate an optimal gain value from this data have been made (Parmar \& Patton, 2015). However, there is no claim or suggestion on how this work transfers to other tasks in general, or to more complex tasks applying spatio-temporal error amplification.

In summary, we wanted to investigate our hypothesis that subjects can achieve better performance in accurately reproducing a fast-paced trunk-arm rowing movement when exposed to spatio-temporal visual error amplification during training. Due to suggestions from literature that error amplification is more effective for skilled subjects, and that naïve subjects could be overwhelmed, we decided to investigate non-naïve subjects, which already received one day of training without error amplification. To our knowledge, we are the first investigating spatio-temporal visual error amplification on learning a fast, multidimensional, and cyclic sports movement.

\section{Methods}

\section{Participating subjects}

A total of 20 subjects ( 6 females, 14 males, mean age 25.3 years, SD 2.6 years) were recruited, mainly from the university (students). The subjects were healthy, had normal hearing and normal or corrected-to-normal vision. All subjects had no prior experience with the task and confirmed to be non-rowers and to do at least half an hour of sport per week. All subjects signed an informed consent following the guidelines of the ETH Zurich Ethics Commission, which had approved the study (EK 2014$\mathrm{N}-21$ ).

Five subjects had to be excluded due to technical problems. Two of those subjects dropped out because data recording failed, and baseline data was missing or corrupted. Three subjects dropped out due to hardware failure that could not be resolved during the session.

\section{Apparatus}

This study was performed using our custom-made rowing simulator (Figure 1) (Rauter et al., 2011). This rowing simulator contains a trimmed rowing boat placed in the middle of a Cave Automated Virtual Environment (CAVE). The CAVE is formed by three $4.4 \mathrm{~m}$ times $3.3 \mathrm{~m}$ screens placed in front and on the sides of the boat. The rowing simulator was set up for sweep rowing on portside (left side in direction of travel), meaning the subject manipulated a single oar with both hands traveling backwards.

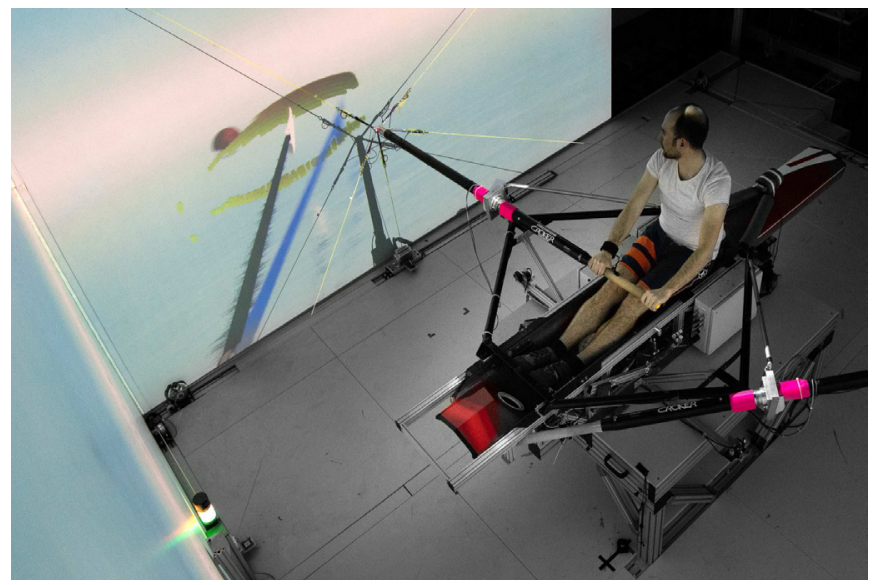

Figure 1: Apparatus with coauthor demonstrating rowing scenario and visual augmented feedback. The depicted individual (coauthor) provided written informed consent for the image's publication.

Sounds were provided through over-ear stereo headphones (with a frequency range of $14 \mathrm{~Hz}$ to $26 \mathrm{kHz}$ ). The provided sounds were rendered oar-water interactions implemented using $\mathrm{C}++$ with an update rate of $30 \mathrm{~Hz}$.

Haptics were provided by a tendon-based parallel robot (Rauter, Zitzewitz, Duschau-Wicke, Vallery, \& Riener, 2010). The parallel robot was controlled by a Matlab/Simulink ${ }^{\circledR}$ (r2013b, MathWorks, MA, USA) model on an xPC-target at a fixed update rate of $1 \mathrm{kHz}$. The haptics displayed by the robot were either forces of the rendered water resistance, or forces of a position control mode during instruction, where the robot completely controls the oar position and guides the subject. Water resistance was realized with a virtual rowing model (Rauter et al., 2010), which allowed subjects to accelerate and decelerate the boat in the virtual environment by interacting with the oar in a natural manner. Rowing simulation and water resistance realism were confirmed with a study showing that training in the simulator fostered skill gains in real rowing on water to a similar extent as real rowing training on water (Rauter et al., 2013).

\section{Task}

Within this study, subjects were trained to perform trunk-arm sweep rowing. In sweep rowing each rower operates one oar with both hands. Synchronized oar-water interaction forces are required to efficiently drive the boat forwards. In our simulator, the subject was rowing on port-side and a virtual boat mate performed a synchronized movement on starboard-side.

Trunk-arm rowing refers to a rowing mode, where the subject keeps legs extended and omits leg push. Trunk-arm rowing is a common training exercise in rowing, for example during warmup or during training sessions with the goal to improve rowing technique.

Trunk-arm sweep rowing stroke is a complex oar movement requiring a coordination of trunk and arm movements. 
Additionally, this complex movement should be synchronized with the boat mates. To account for challenges of such a coordinated and synchronized movement, subjects were instructed to reproduce a reference movement, while focusing on both spatial and velocity profile. Reproducing a desired spatial and velocity profile is especially challenging because of the oar-water interaction: the required forces vary depending on the relative velocity between boat, oar, and water.

Identical to our previous studies (Rauter et al., 2015; Sigrist et al., 2014), subjects were instructed to reproduce a given reference stroke as accurately as possible regarding both spatial and velocity profile of this reference stroke. The reference stroke was prerecorded by a rowing expert at a typical training stroke rate of 24 strokes per minute. The recorded reference was postprocessed, to have a smooth, cyclic trajectory with $C^{2}$ continuity and an exact duration of $2.5 \mathrm{~s}$ reflecting an exact stroke rate of 24 strokes/min. It was further rescaled to a movement range at the oar handle center of $0.67 \mathrm{~m}$ horizontally (corresponds to $44^{\circ}$ horizontal oar angle at the oarlock) and $0.19 \mathrm{~m}$ vertically (corresponds to $12.5^{\circ}$ vertical oar angle at the oarlock) to ensure a reference movement that could be performed by subjects taller than the minimally requested $1.65 \mathrm{~m}$ (inclusion criteria). Oar blade rotation and leg movements were not included in the task, the oar blade was instructed to be kept in vertical orientation only and the rowing seat was fixed at a position, where the subject's legs were extended.

Our training task can be classified as a continuous rhythmic motor task (Marchal-Crespo et al., 2015), requiring the subjects to perform repetitive continuous movements. "Trajectory tracking task" is another term that is commonly used in motor learning experiments, where a fixed desired reference movement is used. However, in our test conditions there is no augmented feedback or reference displayed that the subjects could track. We would therefore suggest referring to such a task as a "trajectory reproducing task".

\section{Augmented visual feedback and error amplification}

During the test, and non-feedback conditions, no spatial or temporal reference cues except the water level were provided visually. In contrast, during the training conditions the subjects received augmented visual feedback (visible on the left in Figure 1). On top of the rowing simulation that includes visual rendering of water and subject oar, this feedback consisted of a virtual blue reference oar and green to red traces. The blue reference oar was displaying the reference movement but rendered with increasing transparency the smaller the subject's instantaneous angular distance to the current reference position was. It was faded out completely if the instantaneous angular distance between subject's oar and reference was smaller than $4^{\circ}$. The green to red traces were drawn by the subject oar if the spatial deviation from reference trajectory was larger than $3.6^{\circ}$ vertically or $1.9^{\circ}$ horizontally. These traces were drawn in green for small deviations and their colors got increasingly reddish the larger the spatial deviation from the subject oar was to the reference trajectory. The end of the traces was faded out such that only deviations performed during the last $8 \mathrm{~s}$ were displayed. All components of this visual feedback were designed such that the feedback faded out if the subjects performance was near the reference to avoid the subject relying on the feedback and becoming dependent on it (Sigrist et al., 2014). This feedback principle is also referred to as bandwidth feedback introduced by (Sherwood, 1988).

If the subject was not close enough to the current reference position to make it disappear, the instantaneous tracking error could be perceived by the current distance between the subject's oar and the blue reference oar, i.e. a visual spatiotemporal error cue was present. The instantaneous tracking error can be expressed with the following equation:

$\epsilon_{\text {tracking }}(t)=\mathrm{q}_{\text {act }}(t)-\mathrm{q}_{\text {ref }}(t)$,

where $\mathrm{q}_{\text {ref }}(t)$ is the reference oar angular position and $\mathrm{q}_{a c t}(t)$ is the actual subject oar angular position at time $t$. To augment the perceived instantaneous tracking error, we need to either distort the displayed reference oar angular position, the rendered subject oar angular position, or both. We decided to distort the rendered subject oar position, which seems to be the most common approach taken for visual error amplification (Celik et al., 2009; Patton et al., 2013; Sharp et al., 2011; Y. Wei et al., 2005). The subject oar was rendered at an angular position $\mathrm{q}_{E A}(t)$ :

$\mathrm{q}_{E A}(t)=\mathrm{q}_{\text {ref }}(t)+\mathrm{p}_{E A} \cdot \epsilon_{\text {tracking }}(t)$,

where $\mathrm{p}_{E A}$ is the error amplification gain. For $\mathrm{p}_{E A}=1$ the error amplified angular position $\mathrm{q}_{E A}(t)$ and the actual angular position coincide, i.e. there is no visual error amplification. For $p_{E A}>1$ the error is visually amplified and for $\mathrm{p}_{E A}<1$ visual error reductions could be realized. For our study, the parameter $p_{E A}=1.6$ was chosen for the error amplification. This choice was based on a pilot study with 6 uninformed subjects (Basalp et al., 2016).

A drawback of such a spatio-temporal error amplification was that the error changes over time. Therefore, the displayed subject oar on the screen would move even if the subject did not move the physical oar. Since this visual error amplification was consciously perceivable during training, we decided to inform subjects of the VisualEA group when they will be exposed to error amplification. They were explained how the error amplification works by displaying a distorted oar position, and that it only amplifies errors that they perform themselves. This instruction was given to ensure that they feel responsible for the errors they get displayed, such that they do not hold the robot, the setup, a changed reference, or broken sensors accountable.

Thus, we assumed that a gain of $\mathrm{p}_{E A}=1.6$ would be a reasonable choice for our movement and setup, since it was the smallest gain that was clearly making a difference in our pilot study. We expected that the non-naive subjects informed about the presence of error amplification could cope with this gain. 


\section{Definitions}

To avoid misunderstandings due to terminology in the multidisciplinary field of robot-assisted motor-learning, we will use the following definitions within our work:

A Dissimilarity Metric is a quantification of inequality for different entities of the same type. For simple entities, e.g. two points, this can be a simple metric such as distance. However, for more complex entities, such as rowing strokes stored as time series or trajectories, different, more complex dissimilarity metrics are necessary. Both error and variability require a defined dissimilarity metric.

Error is the average dissimilarity between each task repetition (e.g. each rowing stroke) and reference. If the goal is an accurate movement reproduction, high error means low performance. Variability is the average dissimilarity between each task repetition and all other task repetitions. High variability means low consistency.

Learning to perform well means improving (persistently) the ability to achieve higher performance. If a perfect reference is known, learning to perform well is directly related to a reduction of error. Thereby, error is conceptually lower bounded by variability. To achieve an error of zero, all repetitions need to match reference exactly, thus variability would be zero. If the error is not greater than the variability, subjects might have reached a level, where the remaining observed error originates purely from variability. In that case, a reduction of variability would be necessary to further reduce errors. Therefore, learning to perform well may be indirectly related to a reduction of variability.

Learning to perform consistently means improving (persistently) the ability to achieve higher consistency. Learning to perform consistent is directly related to a reduction of variability. Variability is independent of error. To achieve zero variability, all repetitions must be identical, but can be arbitrarily different to the reference. Therefore, learning to perform consistently is not related to error.

\section{Kinematic Evaluation}

Vertical and horizontal oar angles were estimated in the robot's control computer at $1 \mathrm{kHz}$ from the measured tendon lengths. These two oar angles served as minimal coordinates for the kinematic evaluation and were recorded at $100 \mathrm{~Hz}$. Kinematic evaluation was performed in custom-written programs in Matlab $^{\circledR}$ (MathWorks, MA, USA).

The recorded data was first cut into blocks corresponding to the different test, training and non-feedback conditions of the experimental protocol. The oar angles were then segmented into rowing strokes, i.e. movement repetitions, at the point in time that corresponds to the minimal horizontal oar angle of the subject's movement. The first five and the last rowing stroke of each block were excluded from further analysis to avoid the evaluation of transition effects. Subjects needed a couple of strokes to accelerate the boat to achieve a reasonable travel velocity. Rowing strokes with a rate beyond 22 to 26 strokes/min were labeled invalid and excluded from analysis. The remaining rowing strokes and reference were resampled to 250 data points for the analysis.

Two different dissimilarity metrics were applied: spatial dissimilarity and velocity magnitude dissimilarity. Those two metrics were chosen because no absolute timing reference was given during the test conditions and we would not know on how to weight spatial against temporal dissimilarity. Both dissimilarity metrics were applied to compare all strokes of a condition against reference (for error) and against all other strokes in the same condition (for variability). Thus, we obtained four outcome variables: spatial error, spatial variability, velocity (magnitude) error, and velocity (magnitude) variability.

Spatial dissimilarity was defined using dynamic time warping (Giese \& Poggio, 2000) with a zero weighting of the temporal shifts. Dynamic time warping assigns each sample of a rowing stroke to one sample of either reference (for error) or other strokes (for variability) by minimizing spatial and weighted temporal differences under the constraint of time continuity (causal temporal order of samples). Spatial dissimilarity is then calculated as the average distance between these assigned samples. The benefit of this metric compared to using a fixed temporal assignment (e.g. by the same index) is that small temporal shifts that would have a large impact on spatial dissimilarity are not overrated (Vlachos, Hadjieleftheriou, Gunopulos, \& Keogh, 2003). In other words, a reduction of a spatial dissimilarity based on fixed temporal assignments could be based on improvements of temporal accuracy. In contrary, a reduction of a spatial dissimilarity based on dynamic time warping cannot be based on improvements of temporal accuracy only.

The velocity magnitude dissimilarity was chosen as a measure for temporal accuracy, since for this repetitive trunk-arm rowing absolute temporal constraints are not provided during test conditions. The velocity magnitude dissimilarity was defined using the same dynamic time warping assignment used for the spatial dissimilarity but simplified to align the absolute value of the velocity in one dimension between one stroke and either reference (for error) or other strokes (for variability).

Illustrative examples for this dissimilarity metrics can be found in (Rauter et al., 2015; Sigrist et al., 2014) for spatial error and velocity magnitude error.

The subject's performance and consistency of a single condition were evaluated with the spatial error $\left(\bar{\epsilon}_{s}\right)$, spatial variability $\left(\bar{\vartheta}_{s}\right)$, velocity magnitude error $\left(\bar{\epsilon}_{v}\right)$, and velocity magnitude variability $\left(\bar{\vartheta}_{v}\right)$ using all valid strokes in this test condition.

The main benefit of using the same dissimilarity metric for evaluating both error and variability is that those two outcomes can then be directly related quantitatively and not only qualitatively. Using the same evaluation pipeline results in outcomes in the same unit and for our task in the same order of magnitude. If subjects reach low error levels that are eventually lower bounded by their variability this becomes eminent due to similar values of both outcomes. 
In addition to these four main outcomes, the average instantaneous tracking error $\left(\bar{\epsilon}_{\text {tracking }}\right)$ was defined as the average angular distance between reference and subject oar using a fixed temporal assignment. The instantaneous tracking error was only evaluated during the training conditions with feedback, where the reference oar was shown to the subject. It could not be evaluated meaningfully for the test conditions without feedback, where the reference oar was not shown, and the subjects did not receive any cues on absolute timing. The grand average was calculated analogously to the main outcomes, evaluating each valid stroke and then taking the average during a training condition. However, the individual strokes were not down-sampled to 250 data points for the instantaneous tracking error evaluation.

\section{Experimental Protocol}

Subjects were invited to the laboratory on three consecutive days. The study was held in a parallel design of two groups: VisualEA and Contro/Visual. The 15 included subjects were assigned to the VisualEA group ( 3 females, 5 males, 23-26 years, mean age 25.3 years, SD 2.6 years) and the Contro/Visual group (3 females, 4 males, 19-27 years, mean age 25.1 years, SD 3.3 years).

Both groups received the same training on day 1 , such that on day 2 the effect of error amplification could be tested on two comparably skilled and non-naïve groups of subjects. The assignment of subjects to groups was done in a random, gendermatched, pairwise filling up of both groups, e.g. the first male subject was assigned to a group by coin toss, the next male subject would be assigned to the other group. This process was repeated for each new starting pair of subjects.

On day 1 , general instructions were given, and one investigator explained the handling, safety features, and remaining risks of the simulator. After the general instruction, the investigator demonstrated the use of the rowing simulator by sitting in the simulator and having the robot guide the reference movement with a position controller for $\sim 30 \mathrm{~s}$. This short demonstration was performed to give the subjects an overview of the interaction with the simulator and the movement range and velocities. Following this general introduction and demonstration, every subject received a $180 \mathrm{~s}$ instruction (corresponds to 72 reference strokes), where the robot fully guided them through the reference movement with position control. Subjects were instructed to memorize spatial and temporal characteristics of the reference movement, since they would be requested to reproduce the reference movement as accurately as they can in the following baseline test. No strategy on how to benefit from the robot-guided reference movement was instructed. Head orientation of the subjects was not controlled for, i.e. they were free to watch the oar and feedback with their head turned to the right, or to watch straight ahead and only occasionally gaze at the feedback and their oar.

A $180 \mathrm{~s}$ baseline test (BL) followed the instruction, where the subjects rowed without robotic guidance (Figure 2). The subjects had to reproduce the movement on their own, based on their memory of the instruction. In baseline and all subsequent non-feedback and test conditions only the task rendering, but no augmented feedback was displayed to the subject. Task rendering consisted of the visually displayed ocean scenario, rowing boat and subject oar, the auditorily displayed purling sound of the subject oar on the right headphone speaker, and the haptically displayed oar-water interaction forces.

\begin{tabular}{|c|c|c|c|c|c|c|c|c|c|c|c|}
\hline \multirow[t]{2}{*}{ day 1} & $\begin{array}{l}3 \\
1 \\
\end{array}$ & $\begin{array}{l}3^{\prime} \\
\perp \\
\end{array}$ & $\begin{array}{l}1 \\
1 \\
\end{array}$ & $\begin{array}{l}3 \\
1 \\
\end{array}$ & $\begin{array}{l}1 \\
1 \\
\end{array}$ & $\begin{array}{l}3 \\
1 \\
\end{array}$ & $\begin{array}{l}1^{\prime} \\
1\end{array}$ & $\begin{array}{l}3 \\
1 \\
\end{array}$ & $1^{\prime}$ & $\begin{array}{l}3 \\
\perp \\
\end{array}$ & $\begin{array}{l}1 \\
\perp \\
\end{array}$ \\
\hline & BL & TR1 & NF1 & TR2 & NF2 & TR3 & NF3 & TR4 & NF4 & TR5 & NF5 \\
\hline & $\begin{array}{l}3 \\
\end{array}$ & $3^{\prime}$ & $1^{\prime}$ & $3^{\prime}$ & 1 & $3^{\prime}$ & $1^{\prime}$ & $3^{\prime}$ & $1^{\prime}$ & $3^{\prime}$ & $\begin{array}{l}1^{\prime} \\
1\end{array}$ \\
\hline & RE2 & TR6 & NF6 & TR7 & NF7 & TR8 & NF8 & TR9 & NF9 & TR10 & $\mathrm{NF1C}$ \\
\hline & $\begin{array}{c}3^{\prime} \\
1 \\
\text { RE3 }\end{array}$ & & & & & & & & & & \\
\hline
\end{tabular}

Figure 2: Protocol: $3^{\prime}$ and $1^{\prime}$ denote the duration in minutes of the condition. BL denotes baseline. TR1-TR5 denotes training conditions with feedback without error amplification and TR5-TR10 training conditions with feedback and error amplification for the VisualEA group. NF1-NF10 denotes no feedback conditions. RE2 and RE3 denote retention tests on day 2 and 3 respectively. A short break of $25 \mathrm{~s}$ was scheduled between the conditions on one day.

After the baseline test, both groups received the same five training sessions, which each consisted of a $180 \mathrm{~s}$ (corresponds to 72 reference strokes) training condition (TR1-TR5) with the visual augmented feedback without error amplification $\left(\mathrm{p}_{E A}=1.6\right)$ and a $60 \mathrm{~s}$ (corresponds to 21 reference strokes) no-feedback test condition (NF1-NF5). Before the first training condition with visual augmented feedback, the feedback was described to the subject and a familiarization period of maximum $60 \mathrm{~s}$ was provided additionally. Training condition and no-feedback test conditions were separated with a break of $\sim 25 \mathrm{~s}$.

On day 2, the subjects started with a retention test (RE2) of 180 s. After RE2, five training sessions consisting of 180 s augmented visual feedback training (TR6-TR10) and $60 \mathrm{~s}$ no-feedback test conditions (NF6-NF10) were performed. In those five training conditions of the second day, the VisualEA group received augmented visual feedback with error amplification and a fixed gain of $\mathrm{p}_{E A}=1.6$, while the ControlVisual group received the augmented visual feedback without error amplification $\left(\left(\mathrm{p}_{E A}=1\right)\right.$ again.

On day 3 , only the final retention test (RE3) of $180 \mathrm{~s}$ was performed.

To avoid effects on performance caused by a speed-accuracy trade-off, the subjects' stroke rate was controlled by the operator to be in the range of 22 to 26 strokes/min during all test conditions (baseline test, retention tests, and no-feedback tests). If a subject was rowing with a higher or lower stroke rate, the operator would instruct verbally to increase or decrease the stroke rate. During the training conditions, the stroke rate was not controlled by the operator and the subjects were free to interact with the augmented visual feedback. 


\section{Statistical Evaluation}

Statistical evaluation was performed in Matlab ${ }^{\circledR}$, and independently for all four main outcome metrics: spatial error $\left(\bar{\epsilon}_{s}\right)$, spatial variability $\left(\bar{\vartheta}_{s}\right)$, velocity magnitude error $\left(\bar{\epsilon}_{v}\right)$, and velocity magnitude variability $\left(\bar{\vartheta}_{v}\right)$.

One-way ANOVA was used to check for significant differences between the groups at baseline (BL). Violations for the assumption of equal variance were tested with Levene's test. If the assumption of equal variance was violated a nonparametric Kruskall-Wallis test was performed instead of a parametric one-way ANOVA.

A $2 \times 2$ repeated-measures ANOVA was performed for the main effect of test (BL, RE2) and the interaction between group (VisualEA, ControlVisual) and test. This repeated-measures ANOVA should ensure that both groups advanced to a nonnaïve and comparable skill level over the first day of training. A separate $2 \times 2$ repeated-measures ANOVA was performed for the main effect of test (RE2, RE3) and the interaction between group (VisualEA, ControlVisual) and test. This separate repeatedmeasures ANOVA should asses if subjects did advance over the second day of training and assess persistent effects of the visual error amplification.

The main outcomes were also investigated during trainings on the second day using a $2 \times 5$ repeated-measures ANOVA for the main effect of training (TR6, TR7, TR8, TR9, TR10) and the interaction between group (VisualEA, ControlVisual) and training. Violations of sphericity were tested for with Mauchly's test for sphericity, and if necessary Greenhouse-Geissner correction was applied. In addition to the four main outcomes, the same during training repeated-measures ANOVA was conducted independently for the evaluation of the average instantaneous tracking error $\left(\bar{\epsilon}_{\text {tracking }}\right)$.

An additional one-way ANOVA on the instantaneous tracking error $\left(\bar{\epsilon}_{\text {tracking }}\right)$ averaged for each subject over the five trainings of the second day was performed to assess if the instantaneous tracking error was generally lower while error amplification was present.

Besides these outcomes, one-way ANOVA between the groups was used to compare the subject-average number of valid strokes over both the 3-minute test conditions and the trainings of the second day. These tests should ensure that our results are not masked by different numbers of rowing strokes successfully performed at the desired stroke rate.

The significance level was defined as $\alpha=0.05$. Additionally, all p-values between $0.05<\mathrm{p} \leq 0.1$ were reported as trending towards significance.

\section{Results}

To allow an easier understanding of the used outcome metrics spatial error $\bar{\epsilon}_{s^{\prime}}$ spatial variability $\bar{\vartheta}_{s^{\prime}}$ velocity error $\bar{\epsilon}_{v^{\prime}}$ and velocity variability $\bar{\vartheta}_{v^{\prime}}$, the development of them over all conditions of our experimental protocol and the kinematic data of the main test conditions are illustrated for a single subject in Figure 3.

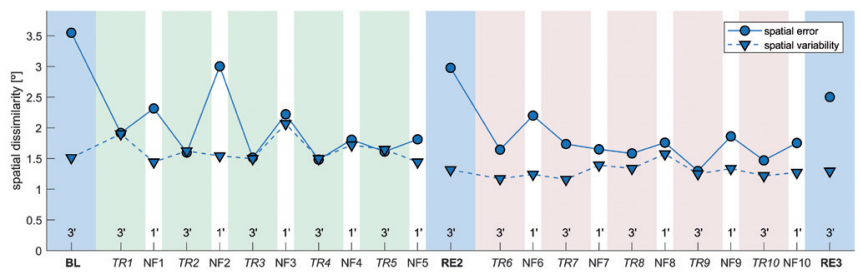

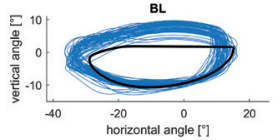
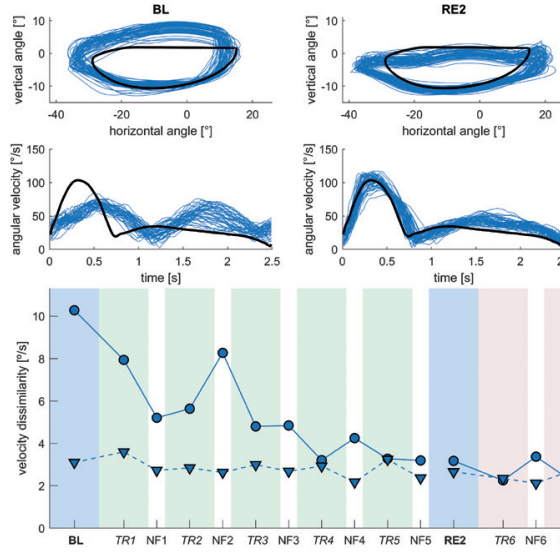

REs
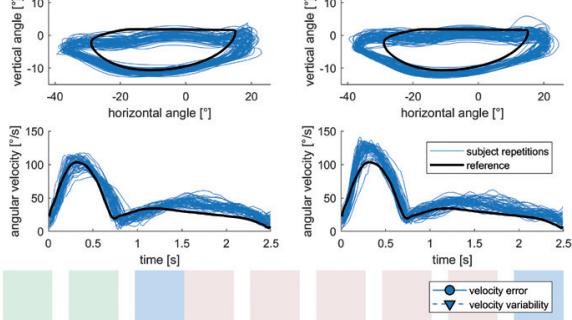

Figure 3: Example of a single subjects' kinematic evaluation: spatial error and spatial variability (top), velocity error and velocity variability (bottom) plotted over all conditions of the experimental protocol, where $\mathrm{BL}$ denotes baseline, TR1-TR10 training conditions with feedback, NF1-NF10 no feedback conditions, $\mathrm{RE} 2$ retention on day 2 , and $\mathrm{RE} 3$ retention on day 2. For the main test conditions $B L, R E 2$, and RE3 the reference movement versus the subjects performed movement repetitions are shown in the middle to illustrate the meaning of error and variability metrics of the applied kinematic evaluation.

Group differences at baseline and in average number of valid strokes

No significant group differences or trends resulted at baseline and there were no group differences or trends in the average number of valid strokes (stroke rate between 22 and 26 strokes/min).

\section{Learning from baseline to retention test on day 2}

Repeated-measures ANOVA revealed a significant main effect of test for all main outcome variables, showing a decrease of all main outcome variables along the tests (Figure 4, Table 1). No significant interactions between group and test were revealed for any variable. 


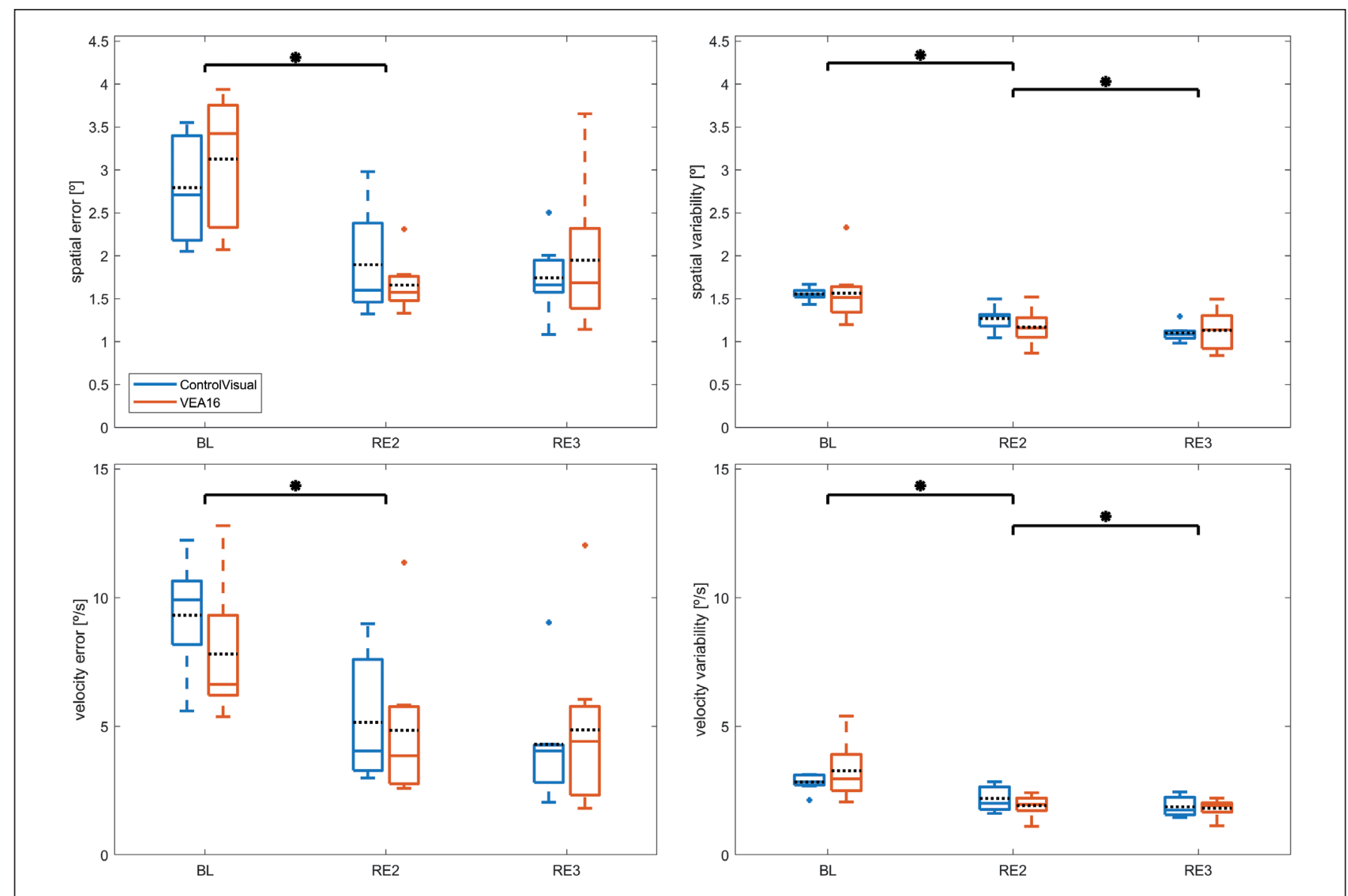

Figure 4: Main outcomes time-course over each test condition for all subjects of the two groups (8 in ControlVisual, and 7 in VisualEA): spatial error (upper left), spatial variability (upper right), velocity error (lower left), and velocity variability (lower right). The boxes in group color denote median and the $50 \%$ coverage intervals. The black dashed line plotted over the boxes is the group mean. The whiskers indicated \pm 2.7 standard deviations or $99.3 \%$ coverage intervals. Small plus symbols denote outliers. Black starred bars denote that the main effect of test in the respective two by two repeatedmeasures ANOVA was significant.

Table 1: Repeated-measures ANOVA results.

\begin{tabular}{|c|c|c|c|c|}
\hline \multirow[t]{2}{*}{ Variables } & \multicolumn{2}{|l|}{ BL to RE2 } & \multicolumn{2}{|l|}{ RE2 to RE3 } \\
\hline & group interaction $F^{g}$ & $p$ & group interaction $F^{g}$ & $p$ \\
\hline \multirow[t]{2}{*}{ Spatial Error } & $F_{1,13}=56.712^{*}$ & $<.001$ & & \\
\hline & $F_{1,13}^{g}=3.305$ & .092 & $F_{1,13}^{g}=3.143$ & .100 \\
\hline Spatial Variability & & & $F_{1,13}^{g}=4.095$ & .064 \\
\hline Velocity Error & $F_{1,13}=67.460^{*}$ & $<.001$ & & \\
\hline Velocity Variability & $F_{1,13}=25.746^{*}$ & $<.001$ & $F_{1,13}=7.4171^{*}$ & .017 \\
\hline
\end{tabular}


Learning from retention test on day 2 to retention test on day 3

No significant main effect resulted for spatial error and velocity error. However, a significant main effect resulted for both spatial variability and velocity variability over the second day of training (Figure 4, Table 1). No significant interactions between group and test were revealed for any variable over the second day of training.

\section{Group differences during trainings of the second day}

Repeated-measures ANOVA during trainings TR6-TR10 revealed a significant main effect of training for velocity variability and a trend for spatial variability (Figure 5, Table 2). These main effects indicated a reduction of variability over the five trainings. A trend towards a significant increase over training was found for velocity error (Table 2). No significant interactions between group and training were revealed for any variable.
Table 2: During training repeated-measures ANOVA results.

\begin{tabular}{lll}
\hline Variables & \multicolumn{2}{c}{ During Training } \\
\cline { 2 - 3 } & main effect $F$ & $p$ \\
\hline Spatial Variability & $F_{4,48}=2.659^{\mathrm{m} 1}$ & $.089^{\mathrm{G}}$ \\
Velocity Error & $F_{4,48}=2.358$ & .067 \\
Velocity Variability & $F_{4,48}=2.766^{*}$ & .038 \\
\hline
\end{tabular}

.${ }^{\mathrm{m} 1}$ Mauchly Violation with $\chi^{2}(2)=18.833, p=.027$

. ${ }^{G}$ Greenhouse-Geissner corrected $p$-value reported.

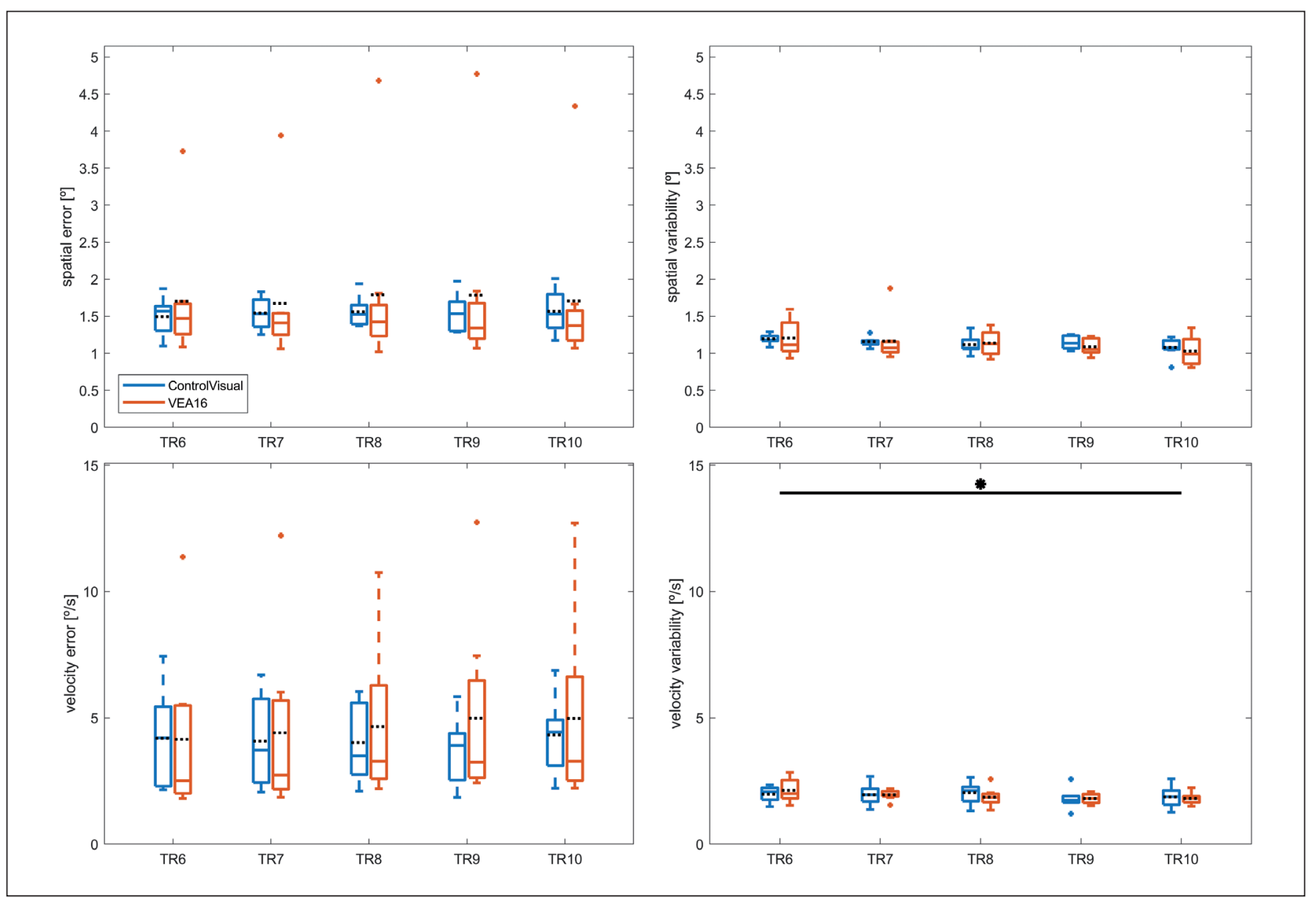

Figure 5: Main outcomes during trainings with or without error amplification (8 in ControlVisual, and 7 in VisualEA) during the trainings of day 2 (TR6-TR10). Black starred bars denote that the respective main effect of training was significant. 
No significant or trending main effects or interactions between group and training resulted from the repeated-measures ANOVA for the instantaneous tracking error (Figure 6). The instantaneous tracking error averaged for each subject over the five trainings of the second day did not result in a significant group difference.

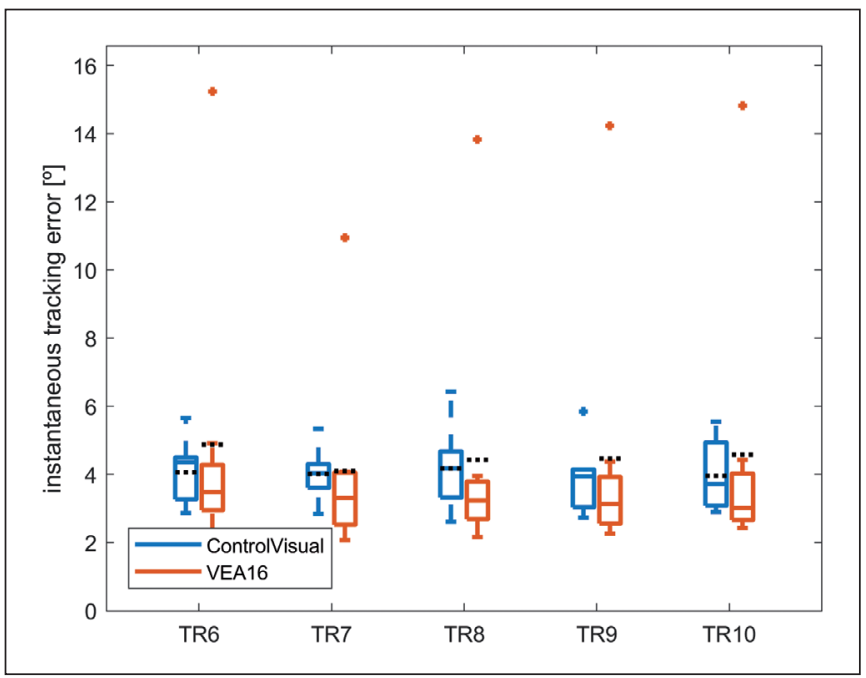

Figure 6: Instantaneous tracking error during training with or without error amplification (8 in ControlVisual, and 7 in VisualEA) during the trainings of day 2 (TR6-TR10)..

\section{Outlier in visual error amplification group}

One very exceptional participating subject from the VisualEA group reached very high error values during training conditions (Figure 5, left and Figure 6). Our complete statistical evaluation was repeated under the exclusion of this subject.

The results of the second evaluation differed slightly in effect size and $\mathrm{p}$-values but did not change the outcome. Results that were significant, trending towards significance or had a $p>0.1$ stayed the same for all but two results. One unimportant change was that the trend towards group test interaction on spatial error between RE2 and RE3 (Table 1) vanished. The second change was that one-way ANOVA testing the average instantaneous tracking error over all training conditions on the second day now resulted in a trend $\left(F_{1,13}=3.801, p=.075\right)$ towards a significant group difference.

\section{Discussion}

No variable showed a trend or significant difference between the groups at baseline. The subjects significantly reduced all error and variability variables from baseline (BL) to the retention test of the second day (RE2) without any significant group interactions. Therefore, we achieved to provide two comparable groups of non-naïve subjects to compare trainings with and without visual error amplification on the second day of training. The main hypothesis that visual error amplification increases effectiveness for non-naïve subjects could not be confirmed: The missing significant group test interactions in the repeated-measures over the second day of training (Table 1, right) indicate that the VisualEA group did not reduce their errors or variability different than control. We can only conclude that if existent, positive or negative effects on training efficacy from visual error amplification that persist in retention were too small to be observed.

Visual error amplification directly amplifies feedback resolution. Therefore, we assumed subjects of the VisualEA group to experience an increased error awareness, which could result in a better performance during training. However, the VisualEA group did not show lower spatial or velocity errors than control during training. The median of the instantaneous tracking was lower in each training for the VisualEA group than in the ControlVisual group (Figure 6). However, we can support this effect only with a trend $(p=.075)$ towards significant group differences in our one-way ANOVA under removal of the outlier subject.

We hypothesized that an increased error awareness could also cause subjects being less satisfied with their performance and therefore exploring more different strategies of movement planning or combinations of sub-movements. While such an increased exploration during training would not result in higher performance, it should be observable in higher variability during training. However, the VisualEA group did not show higher variability than control during training.

It was unexpected that we could not find any significant differences in performance or variability during trainings with or without error amplification. Subjectively, the experience of our visual feedback with error amplification was more demanding than without, and our pilot subjects had shown very distinct behavior (Basalp et al., 2016).

\section{Potential limitations of our experimental protocol}

The high between-subject variability during training could have masked any potential differences during training. Subjects were instructed to find their own strategy to benefit from the feedback and to pursue the goal of being able to reproduce the reference movement as accurate as possible in later test conditions. Evaluation of training conditions must be taken with care. Subjects did not necessarily try to perform as good as possible during their trainings. Additionally, learning complex movements seems to introduce additional sources for between subject variability: Besides individual capabilities of understanding the feedback and learning to perform, the subjects' ability to find and chose successful strategies to benefit from a feedback seem to become increasingly important. One out of seven subjects in our VisualEA group already completely failed to benefit from the feedback, which seems to be an illustrative observation supporting this assumption. To counteract the increased between subject variability, an increase of the sample size would be the obvious strategy. However, performance during the test conditions did not even indicate an insignificant 
beneficial effect (Figure 4), therefore an increase of the sample size was not considered worthwhile.

Subjects might have reached their maximum achievable performance during training with both feedbacks. However, we know that the similarity metrics and measurement precision were not flooring. Our measurements and kinematic evaluation is more sensitive, e.g. if position-controlled trial from the robot is measured and evaluated. Further, spatial and velocity errors were higher than spatial and velocity variability, respectively (Figure 4). Therefore, we do not assume that subjects were limited by their capability of performing consistently. Despite having no limitation from technical side or consistency of their own movements, they could still be limited by their ability to accurately reproduce an expert movement. However, we considered the high inter-subject variability during training in both velocity error and spatial error unlikely to purely reflect differences in maximum achievable performance between the subjects.

Subjects were informed and aware of the present error amplification. Therefore, they may have attributed the perceived error partly to the error amplification instead of to themselves. Therefore, although perceiving remaining errors during training, they might have been satisfied with their performance and stopped trying to perform better.

Besides limitations in maximum performance or motivation, subjects might have been limited by their error correction capabilities. The displayed error information can only be used for correcting subsequent rowing strokes due to the fast pace of the task. Performing corrective actions such that they do not cause further kinematic errors along a rowing stroke is challenging due to the water dynamics. Eventually, the non-naïve subjects were not familiar enough to the task dynamics, while more experienced rowers could potentially benefit from the higher resolution feedback provided by the error amplification.

\section{Potential limitations of our implementation of error amplification}

The trainings from VisualEA and ControlVisual might have been too similar, e.g. a static error amplification gain of 1.6 was too low to make a large enough difference. However, since our five pilot subjects have failed to train with a gain of 1.8 , it is unclear if higher static gains would provide an improvement. Instead of a static gain error amplification, error offsetting or using a gain with a dynamic adaptation to performance might be more promising solutions, which could also help preventing that some subjects fail completely when training with error amplification.

Error offsetting, e.g. applied in (Celik et al., 2009; Patton et al., 2013; Y. Wei et al., 2005), would augment errors with an offset $\epsilon_{b l}$ instead of amplifying the actual error. The offset is commonly a function of the error profile at an earlier test condition, e.g. baseline, $\epsilon_{\text {off }}=\epsilon+\epsilon_{b l}$. Error offsetting achieved slightly more promising results than error amplification in terms of more or faster error reduction (Celik et al., 2009; Patton et al., 2013; Y. Wei et al., 2005). However, the lowest absolute tracking error was achieved by the group with error amplification (Celik et al.,
2009). Nevertheless, we consider testing the transfer of error offsetting to complex movements an interesting topic for future research.

Progressive error amplification, where gains are either adapted as a function of performance of previous repetitions and of a forgetting factor (Emken, Benitez, \& Reinkensmeyer, 2007), or faded over time (Crespo \& Reinkensmeyer, 2008), would fit well as an opposing concept to assist-as-need in guidance. Recently, an approach was presented for haptic error augmentation, where error probability fields were applied to amplify often reoccurring errors stronger than rare ones (Fisher et al., 2015). We consider adaptive error amplification a promising concept for future research to avoid overwhelming subjects while challenging them at an individual level. However, adaptive gain or more advanced error augmentation strategies increase implementation complexity and require setting of more parameters. Currently, experience on how to tune these additional parameters for fast-paced sports movements seem to be missing.

Using spatio-temporal error amplification visually may also be a critical design choice responsible for the null result. Studies reporting beneficial effects of spatio-temporal error amplification are sparse, and studies reporting beneficial outcomes were applying haptic error amplification (Abdollahi et al., 2013), or a combination of haptic and visual error amplification (Rozario et al., 2009). However, for our trunk-arm rowing task, haptic error augmentation turned out to be impractical. During trunk-arm rowing the oar handle is moved closely to the subjects' chest, trunk, and legs. High error augmentation forces could drive the oar handle to collide with the human and therefore are a safety risk. Scaling down the error augmentation to low but safe force magnitudes resulted in forces that were not consciously perceivable due to the high forces of rendered water resistance, friction, and Coriolis effects.

Using a visual error amplification that only amplifies spatial error might be easier to transfer. However, a purely spatial error amplification does not affect errors in temporal features, e.g. velocity profiles. Therefore, we would expect such a transfer to be of less relevance for complex movements, where temporal features are a fundamental aspect.

\section{Potential limitations of visual error amplification for complex} movements

Another possible explanation for the lack of differences could be that error-driven learning is not the dominant mechanism for the learning of our trajectory reproducing task. A recent literature review focusing on haptic assistance during motor learning from a neuro-cognitive perspective found that observational learning would be the primary mechanism for trajectory learning of both spatial and dynamic properties (Heuer \& Lüttgen, 2015). This finding might hold as well for visual feedback. In our experiment both groups received correct visual spatio-temporal reference cues with the movement of blue reference oar, and additionally correct visual spatial reference by locations where no error traces were drawn. Regarding observational learning, 
the visual feedback provided the same cues for both groups. Therefore, the group differences might be missing because the subjects mainly consider and memorize the correct reference cues instead of the error between their own position and reference. The alternative visual error amplification strategy to cheat the subjects on the reference position instead of their current position would not have the same problem of giving the groups the same correct reference information. However, assuming observational learning was dominant, giving distorted reference would likely lead to decreased effectiveness of training. According to that theory, cheating the subject on the reference position would not be a promising strategy to increase training effectiveness in our task either.

Even though error amplification has been shown to be beneficial for skilled subjects in literature (Hasson et al., 2016; Marchal-Crespo et al., 2014; Milot et al., 2010), we were not able to show beneficial effects of visual error amplification with the chosen feedback for non-naïve subjects in our trunk-arm rowing task. If beneficial effects of error amplification are transferable from simple movements to fast and complex sports movements with other error augmentation strategies stays an open question. However, since error amplification seems to be very susceptible to implementation details for which an informative basis is missing, the practical use of error amplification in sports training remains questionable.

\section{Conclusion}

To our knowledge, we were the first who performed a study applying visual error amplification to train non-naïve subjects learning to reproduce a realistic fast sports movement with a fading visual feedback. Additionally, we have provided a definition per which movement performance and variability can be evaluated, such that they are consistent with each other and can be quantitatively related. We also provided an example implementation with our outcome metrics and an example application with our study.

We have not found any relevant effects of error amplification applied to a fading, visual feedback for non-naïve subjects performing a realistic rowing movement. Even though a trend towards lower instantaneous tracking error was observed during training, no positive learning effects could be observed in test conditions.

Possible reasons or explanations for this null result are speculative. While it might hold true that spatio-temporal error amplification is not transferrable to complex tasks at all, also different design decisions could be responsible for the missing effects. The main conclusion that we draw is that a realization of a beneficial error amplification strategy for complex movements remains questionable. The added complexity when investigating complex movements requires design decisions for which an informative basis is missing. To conclude if error amplification may or may not enhance the learning of complex movements further studies are required.

\section{Acknowledgements}

Special thanks go to Prof. Dr. Georg Rauter for his previous work and support enabling this study, as well as for his constant readiness to assist us with council or technical issues. We want to thank Dr. Laura Marchal-Crespo and Dr. Jaime Duarte for the valuable discussions and their feedback to our work. Further, we want to thank Michael Herold-Nadig for his technical support and safety testing. Finally, we want to thank Patrick Seeburger for his great assistance and motivation during the measurements, and all the subjects for their voluntary participation.

\section{Funding}

This work was supported by the Swiss National Science Foundation [SNF Grant "Acceleration of complex motor learning by skill level-dependent feedback design and automatic selection", CR2312_152817], and Clinical Research Priority Program of the University of Zurich [CRRP "Neuro-Rehab"].

\section{Competing Interests}

The authors have declared that no competing interests exist.

\section{Data Availability Statement}

All relevant data are within the paper.

\section{References}

Abdollahi, F., Lazarro, E. D. C., Listenberger, M., Kenyon, R. V., Kovic, M., Bogey, R. A., Hedeker, D., et al. (2013). Error Augmentation Enhancing Arm Recovery in Individuals With Chronic Stroke A Randomized Crossover Design. Neurorehabilitation and neural repair, 1545968313498649.

Basalp, E., Gerig, N., Marchal-Crespo, L., Sigrist, R., Riener, R., \& Wolf, P. (2016). Visual Augmentation of Spatiotemporal Errors in a Rowing Task. 11th joint dvs Conference on Motor Control \& Learning, Biomechanics \& Training, 2016, Germany. doi: 10.3929/ethz-a-010799461

Bouchard, A. E., Corriveau, H., \& Milot, M.-H. (2015). Comparison of haptic guidance and error amplification robotic trainings for the learning of a timing-based motor task by healthy seniors. Frontiers in systems neuroscience, 9.

Celik, O., Powell, D., \& Malley, M. K. (2009). Impact of visual error augmentation methods on task performance and motor adaptation. Rehabilitation Robotics, 2009. ICORR 2009. IEEE International Conference on (pp. 793-798). IEEE. 
Crespo, L. M., \& Reinkensmeyer, D. J. (2008). Haptic guidance can enhance motor learning of a steering task. Journal of motor behavior, 40(6), 545-557.

Duarte, J. E., \& Reinkensmeyer, D. J. (2015). Effects of robotically modulating kinematic variability on motor skill learning and motivation. Journal of neurophysiology, 113(7), 2682-2691.

Emken, J. L., Benitez, R., \& Reinkensmeyer, D. J. (2007). Humanrobot cooperative movement training: Learning a novel sensory motor transformation during walking with robotic assistance-as-needed. Journal of neuroengineering and rehabilitation, 4(1), 16. doi: 10.1186/1743-0003-4-8

Emken, J. L., \& Reinkensmeyer, D. J. (2005). Robot-enhanced motor learning: accelerating internal model formation during locomotion by transient dynamic amplification. IEEE Transactions on Neural Systems and Rehabilitation Engineering, 13(1), 33-39. doi: 10.1109/TNSRE.2004.843173

Fisher, M. E., Huang, F. C., Klamroth-Marganska, V., Riener, R., \& Patton, J. L. (2015). Haptic error fields for robotic training. World Haptics Conference (WHC), 2015 IEEE (pp. 434-439). doi: 10.1109/WHC.2015.7177750

Giese, M. A., \& Poggio, T. (2000). Morphable models for the analysis and synthesis of complex motion patterns. International Journal of Computer Vision, 38(1), 59-73.

Hasson, C. J., Zhang, Z., Abe, M. O., \& Sternad, D. (2016). Neuromotor Noise Is Malleable by Amplifying Perceived Errors. PLOS Computational Biology, 12(8), 1-28. doi: 10.1371/journal.pcbi.1005044

Heuer, H., \& Lüttgen, J. (2015). Robot assistance of motor learning: A neuro-cognitive perspective. Neuroscience \& Biobehavioral Reviews, 56, 222-240.

LaViola Jr, J. J. (2000). A discussion of cybersickness in virtual environments. ACM SIGCHI Bulletin, 32(1), 47-56.

Marchal-Crespo, L., Lopez-Oloriz, J., Jaeger, L., \& Riener, R. (2014). Optimizing learning of a locomotor task: amplifying errors as needed. 2014 36th Annual International Conference of the IEEE Engineering in Medicine and Biology Society (pp. 5304-5307). IEEE.

Marchal-Crespo, L., Schneider, J., Jaeger, L., \& Riener, R. (2014). Learning a locomotor task: with or without errors? Journal of neuroengineering and rehabilitation, 11(1), 1.

Marchal-Crespo, L., Wolf, P., Gerig, N., Rauter, G., Jaeger, L., Vallery, H., \& Riener, R. (2015). The role of skill level and motor task characteristics on the effectiveness of robotic training: first results. Rehabilitation Robotics (ICORR), 2015 IEEE International Conference on (pp. 151-156). IEEE.

Milot, M. H., Marchal-Crespo, L., Green, C. S., Cramer, S. C., \& Reinkensmeyer, D. J. (2010). Comparison of erroramplification and haptic-guidance training techniques for learning of a timing-based motor task by healthy individuals. Experimental brain research, 201(2), 119-131. doi: 10.1007/s00221-009-2014-z

Parmar, P. N., \& Patton, J. L. (2015). Optimal gain schedules for visuomotor skill training using error-augmented feedback. Robotics and Automation (ICRA), 2015 IEEE International Conference on (pp. 3809-3813). IEEE.
Patton, J. L., Wei, Y. J., Bajaj, P., \& Scheidt, R. A. (2013). Visuomotor Learning Enhanced by Augmenting Instantaneous Trajectory Error Feedback during Reaching. PLoS ONE, 8(1), e46466. doi: 10.1371/journal.pone.0046466

Rauter, G., Sigrist, R., Koch, C., Crivelli, F., Raai, M. van, Riener, R., \& Wolf, P. (2013). Transfer of Complex Skill Learning from Virtual to Real Rowing. PLOS ONE, 8(12), 1-18. doi: 10.1371/ journal.pone.0082145

Rauter, G., Sigrist, R., Marchal-Crespo, L., Vallery, H., Riener, R., \& Wolf, P. (2011). Assistance or challenge? Filling a gap in user-cooperative control. IEEE/RSJ International Conference on Intelligent Robots and Systems (IROS) (pp. 3068-3073). San Francisco, California. doi: 10.1109/IROS.2011.6094832

Rauter, G., Sigrist, R., Riener, R., \& Wolf, P. (2015). Learning of temporal and spatial movement aspects: A comparison of four types of haptic control and concurrent visual feedback. IEEE transactions on haptics, 8(4), 421-433.

Rauter, G., Zitzewitz, J. von, Duschau-Wicke, A., Vallery, H., \& Riener, R. (2010). A tendon based parallel robot applied to motor learning in sports. 3rd IEEE RAS and EMBS International Conference on Biomedical Robotics and Biomechatronics (BioRob), 2010 (pp. 82-87). Tokyo, Japan. doi: 10.1109/ BIOROB.2010.5627788

Rozario, S. V., Housman, S., Kovic, M., Kenyon, R. V., \& Patton, J. L. (2009). Therapist-mediated post-stroke rehabilitation using haptic/graphic error augmentation. Engineering in Medicine and Biology Society, 2009. EMBC 2009. Annual International Conference of the IEEE (pp. 1151-1156). IEEE.

Sharp, I., Huang, F., \& Patton, J. (2011). Visual error augmentation enhances learning in three dimensions. J Neuroeng Rehabil, 8, 1-6.

Sherwood, D. E. (1988). Effect of bandwidth knowledge of results on movement consistency. Perceptual and Motor Skills, 66(2), 535-542.

Sigrist, R., Rauter, G., Marchal-Crespo, L., Riener, R., \& Wolf, P. (2014). Sonification and haptic feedback in addition to visual feedback enhances complex motor task learning. Experimental brain research, 1-17.

Sigrist, R., Rauter, G., Riener, R., \& Wolf, P. (2013). Augmented visual, auditory, haptic, and multimodal feedback in motor learning: A review. Psychonomic Bulletin \& Review, 20(1), $21-$ 53. doi: 10.3758/s13423-012-0333-8

Vlachos, M., Hadjieleftheriou, M., Gunopulos, D., \& Keogh, E. (2003). Indexing multi-dimensional time-series with support for multiple distance measures. Proceedings of the ninth ACM SIGKDD international conference on Knowledge discovery and data mining, KDD '03 (pp. 216-225). Washington, D.C.: ACM. doi: 10.1145/956750.956777

Wang, F., Barkana, D. E., \& Sarkar, N. (2010). Impact of visual error augmentation when integrated with assist-as-needed training method in robot-assisted rehabilitation. Neural Systems and Rehabilitation Engineering, IEEE Transactions on, 18(5), 571-579.

Wei, Y., Bajaj, P., Scheidt, R., \& Patton, J. (2005). Visual error augmentation for enhancing motor learning and 
rehabilitative relearning. 9th International Conference on Rehabilitation Robotics, 2005. ICORR 2005. (pp. 505-510). IEEE.

Wei, Y., Patton, J., Bajaj, P., \& Scheidt, R. (2005). A real-time haptic/graphic demonstration of how error augmentation can enhance learning. Robotics and Automation, 2005. ICRA 2005. Proceedings of the 2005 IEEE International Conference on (pp. 4406-4411). IEEE. 